\title{
Advances in Decentralized Single-Beacon Acoustic Navigation for Underwater Vehicles: Theory and Simulation
}

\author{
Sarah E. Webster \\ Dept. of Mechanical Engineering \\ Johns Hopkins University \\ Baltimore, MD 21218 \\ Email: swebster@jhu.edu
}

\author{
Louis L. Whitcomb \\ Dept. of Mechanical Engineering \\ Johns Hopkins University \\ Baltimore, MD 21218 \\ Email: 1lw@jhu.edu
}

\author{
Ryan M. Eustice \\ Dept. of Naval Arch. and Marine Eng. \\ University of Michigan \\ Ann Arbor, MI, 48109 \\ Email: eustice@umich.edu
}

\begin{abstract}
This paper reports the theory and implementation of a decentralized navigation system that enables simultaneous single-beacon navigation of multiple underwater vehicles. In single-beacon navigation, each vehicle uses ranges from a single, moving reference beacon in addition to its own inertial navigation sensors to perform absolute localization and navigation. In this implementation the vehicles perform simultaneous communication and navigation using underwater acoustic modems, encoding and decoding data within the acoustic broadcast. Vehicles calculate range from the time of flight of asynchronous acoustic broadcasts from the reference beacon. Synchronous clocks on the reference beacon and the vehicles enable the measurement of one-way travel-times, whereby the time of launch of the acoustic signal at the reference beacon is encoded in the acoustic broadcast and the time of arrival of the broadcast is measured by each vehicle. The decentralized navigation algorithm, running independently on each vehicle, is implemented using the information form of the extended Kalman filter and has been previously shown to yield results that are identical to a centralized Kalman filter at the instant of each range measurement. We summarize herein the architecture and design of the acoustic communications (Acomms) system consisting of an underwater acoustic modem, synchronous clock, and the software necessary to run them, and salient results from the validation of the decentralized information filter using a simulated data set.
\end{abstract}

\section{INTRODUCTION}

This paper describes the theory and implementation of a decentralized navigation system that enables simultaneous single-beacon navigation of multiple underwater vehicles. Using underwater modems to combine acoustic communication and navigation, the vehicles employ one-way-travel-time (OWTT) navigation to estimate their own position using ranges from a single georeferenced beacon. The ranges are measured from the one-way travel-time of acoustic broadcasts from the reference beacon and require no centralized processing [4], [6], [27], [28].

The goal of this work is to enable high-precision absolute navigation of multiple underwater vehicles over length scales of $\mathcal{O}(1-100 \mathrm{~km})$. Conventional "dead-reckoning" navigation solutions rely on strap-down sensors such as Doppler velocity $\operatorname{logs}$ (DVLs) and inertial measurement units (IMUs) to measure the vehicle attitude, linear and angular velocity, and acceleration. These measurements can be integrated to estimate relative change in vehicle position but yield an estimate of local displacement with errors that are unbounded over time. In order to achieve bounded-error navigation, additional navigation information is required from an absolute georeferenced source. Traditional methods such as ultra short baseline navigation (USBL) and some implementations of long baseline navigation (LBL) suffer from a lack of scalability where the rate at which multiple vehicles can receive navigation updates decreases linearly as the number of vehicles in the water increases [13]. In addition, LBL navigation requires external, fixed reference beacons that limit the vehicle's navigable range to $5-10 \mathrm{~km}$ from the beacon. In contrast, the acoustic data broadcasts used in OWTT navigation enable the navigation of any vehicle within acoustic range of the reference beacon and the use of a single, moving reference beacon eliminates the need for multiple, fixed beacons and their associated cost and range limitations.

Within the context of single-beacon navigation, the decentralized approach provides a flexible, scalable solution for vehicle navigation. Navigation algorithms that rely on a centralized observer suffer from the severely limited bandwidth and high latency associated with underwater acoustic communication in comparison to typical land-based radio frequency communication networks [14]. Given the speed of sound in water $(\sim 1500 \mathrm{~m} / \mathrm{s})$, transmitting acoustic data over length scales on the order of kilometers results in latency on the order of seconds. And though the bandwidth of acoustic modem technology has increased dramatically in recent years, achieving throughput of up to 2400 bps [23], operationally the average throughput is on the order of 10-50 bps due to the low duty cycle with which these messages are typically transmitted during at-sea operation.

This paper describes the acoustic communication (Acomms) system designed to implement OWTT navigation and the decentralized algorithm developed within the context of OWTT navigation to estimate vehicle position in real-time for multiple vehicles. This paper is organized as follows: Section II provides a brief review of previous work in single beacon navigation and an overview of one-way travel-time single-beacon navigation. Section III describes the acoustic communications system used to implement OWTT navigation including salient results from several field trials. Section IV describes the theory 
and application of the decentralized navigation algorithm developed for OWTT navigation. Section V describes the setup and results of testing the decentralized navigation algorithm with a simulated deep-water data set and Section VI concludes.

\section{Single-Beacon Navigation}

Single-beacon navigation relies on range measurements from a single, georeferenced beacon to provide an absolute position reference. Previous work in the area of single-beacon navigation is extensively reviewed in [27]. This section reviews some of the references most relevant to this paper. Navigation with a single, fixed beacon whose position is known a priori has been reported using several different estimation techniques - a least squares approach in [21] and [1], and a vehicle-based extended Kalman filter (EKF) in [16] and [10]. Navigation with respect to a moving beacon whose position is not known a priori is reported in [17] using a nonlinear least mean square method; in [6] using a maximum likelihood method; and in [27] using a centralized EKF. However, each of these methods, as reported, is only structurally tractable for post-processing, though the authors of [17] suggest improvements that would allow for real-time implementations.

In the OWTT navigation method discussed in this paper, vehicles use the one-way travel time of acoustic messages to measure range. The acoustic messages are broadcast from a single reference beacon that has knowledge of its position in the world frame [4], [6], [27], e.g. a vehicle or ship that is equipped with a GPS receiver. The acoustic broadcasts encode both information about the position of the sender and the time at which the message was transmitted. Each receiving vehicle can then measure the time-of-flight of the acoustic signal using the time-of-launch encoded in the broadcast and the time-of-arrival measured by its own clock. Note that the reference beacon does not need to be stationary as information about the current location of the beacon is included in each broadcast. Between range measurements the vehicle performs dead-reckoning navigation.

In order to measure one-way travel times accurately, the clocks on the reference beacon and the vehicles must be synchronized. The computer of a subsea node is disciplined using a precision clock board that is synchronized to a GPS timeserver prior to the start of the dive, described in more detail below. The reference node is synchronized to a GPS timeserver via the Network Time Protocol (NTP). Synchronized clocks, in addition to enabling OWTT navigation, provide additional advantages during at-sea operations by making it possible to accurately predict acoustic transmissions of other nodes. This enables a time-division multiple access (TDMA) cycle with messages originating at different nodes because they can be scheduled a priori to not overlap. In addition, we are able to maintain a precisely timed, acoustically silent segment of the TDMA to interject messages from the ship if necessary, such as an abort message that commands the vehicle to return to the surface. For a more detailed treatment of OWTT navigation readers are referred to [4], [6], [27], [28].

\section{Acoustic Communications System}

The Acoustic Communications (Acomms) system is a platform-independent system for combined communication and navigation of multiple underwater vehicles that integrates Woods Hole Oceanographic Institution (WHOI) MicroModems [8], [9], a stand-alone software interface [26], and subsea precision clocks [4], [6]. The Acomms system enables both asynchronous communication between multiple underwater and surface vehicles and, when used in conjunction with precision clocks, synchronous communication and navigation. While the communications and one-way travel time features are provided using the WHOI Micro-Modem, the concepts have been developed in a hardware independent framework and can be used with any acoustic system or combination of systems that includes bidirectional communications with synchronous transmission and precision time-tagged reception. The Acomms software, designed to operate symmetrically on all nodes, initializes the modem and issues a sequence of modem commands, defined by the user, to initiate data transmissions between nodes, transmit ranging pings, and interrogate acoustic navigation beacons. In addition, the Acomms software enables the user to specify modem configurations and ensures that the modem stays properly configured in the event of a vehicle or modem reboot. The Acomms software and related hardware have been installed on the Woods Hole Oceanographic Institution vehicles Puma, Jaguar, and Nereus, and have been deployed in sea trials in the North Pacific and South Atlantic. The Acomms system has also been deployed on the two University of Michigan commercial Ocean-Server Iver2 AUV systems [5] and the PPSBoard has been installed on vehicles from the Massachusetts Institute of Technology (MIT) and the Naval Undersea Warfare Center Newport to support OWTT navigation.

\section{A. System Architecture}

The architecture of the Acomms system in a typical twonode setup is depicted in Figure 1, where the vehicle is referred to as the subsea node and the ship is referred to as the topside node. Topside the Acomms software runs on a laptop running Ubuntu Linux and communicates with the ship-board modem via the network using UDP messages through a MOXA ${ }^{\mathrm{TM}}$ serial device server. We use a Meinberg GPS/NTP shipboard timeserver. The Meinberg also supplies a pulse-per-second (PPS) signal to the topside modem, which consists of a $1 \mathrm{~Hz}$ square wave that has its rising edge synchronized with the start of the second. Subsea the Acomms software runs on the main vehicle computer, also running Ubuntu Linux, and communicates with the modem over a serial connection. Acomms communicates with the vehicle's controller and navigation processes over the local network using UDP messages. The time reference on the vehicle is provided by a custom built PPSBoard which uses a SeaScan Inc. temperature compensated crystal oscillator (TXCO) for precision time keeping [4], [6]. The TXCO has a typical drift of $20 \mathrm{~ns} / \mathrm{s}$ which results in a drift of approximately 2.6 meters per day. The PPSBoard with the TXCO provides a PPS signal 


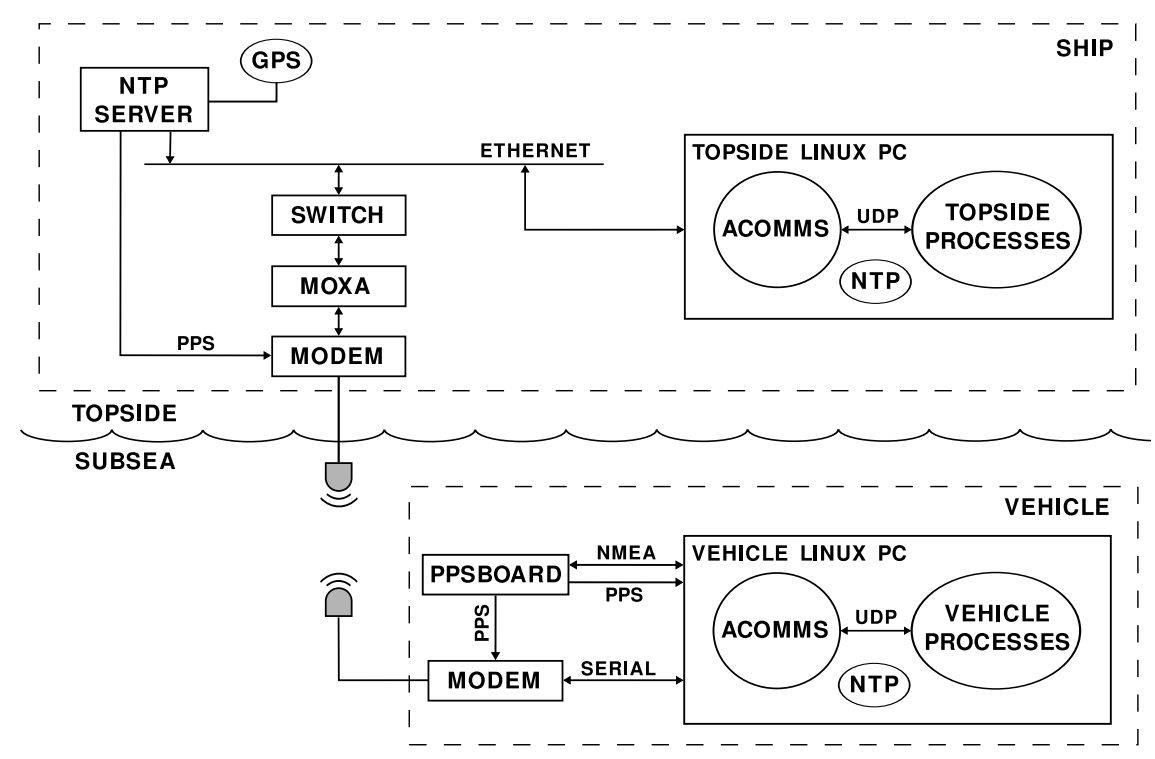

Fig. 1. Typical sea-going architecture for a two-node deployment of the Acomms system.

to the vehicle's modem and provides PPS and NMEA time signals to the vehicle's on-board NTP server.

\section{B. Acomms Hardware}

1) WHOI Micro-Modem: The WHOI Micro-Modem is an underwater acoustic modem capable of encoding data into acoustic data packets that it transmits through the water column [8], [9]. All Micro-Modems are able to receive frequency-shift keyed (FSK) encoded acoustic messages. With the addition of a co-processor board, the Micro-Modems are also able to receive phase-shift keyed (PSK) encoded messages. The Micro-Modem supports 32-bit-long mini-packets and data packets ranging from 32 to 2048 bytes. The range of the Micro-Modem varies with transmit frequency and the acoustic channel characteristics (horizontal/shallow channel versus vertical/deep channel). During recent operations in the Mariana Trench on Nereus, the low data-rate PSK messages were successfully sent and received over the entire length of the $11 \mathrm{~km}$ vertical channel [23].

The Micro-Modem employs its own internal clock to calculate the travel-time of ranging pings and replies from acoustic navigation beacons, and the time-of-arrival of acoustic messages. When the modem is in Synchronous Navigation (SNV) mode, as described in [12], [22], the modem's clock can be synchronized to a PPS signal using a NMEA clock message from the host. Once synchronized, the time-of-arrival (TOA) of each arriving message is reported to have an accuracy of $\pm 125 \mu$ s with respect to the PPS signal [9]. In SNV mode, all transmitted messages are initiated by the modem within \pm $10 \mu \mathrm{s}$ of the rising edge of the PPS signal, referred to as the top-of-the-second [12].

2) PPSBoard: The PPSBoard provides a stable time reference that keeps the undersea vehicle's CPU clock and the vehicle's modem synchronized with the topside clock throughout the mission. The PPSBoard, described in detail in [4] and [6], was developed by the authors Eustice and Whitcomb to provide a free-running, precision timing reference for use subsea that can be synchronized to a GPS timing signal. The PPSBoard supplies a PPS signal and a NMEA-formatted clock message naming the upcoming second to the vehicle computer. The PPSBoard, which is synchronized to a GPS signal while the vehicle is on deck, is used to discipline the vehicle CPU's NTP server. The drift characteristics of the PPSBoard $(\sim 1 \mathrm{~ms}$ drift over 14 hours) ensure that the error introduced in the estimated range between the ship and the vehicle due to the relative drift between the two clocks is small: $\sim 1.5 \mathrm{~m}$ error over 14 hours in the range between the ship and the vehicle. In addition to supplying a timing reference to the host computer, the PPSBoard also supplies a PPS signal to the Micro-Modem to enable its synchronous navigation mode described above.

3) Topside NTP Timeserver: The Meinberg GPS/NTP timeserver, which we use to provide a stable, shipboard timing reference, is a Stratum-1 NTP timeserver. The topside computer stays synchronized with the timeserver over the network via NTP. The Meinberg also supplies a PPS signal to the topside modem. The PPS signal from the Meinberg is accurate to $<1 \mu \mathrm{s}$ [18].

\section{Acomms Software}

The Acomms software is a multi-threaded program written in $\mathrm{C} / \mathrm{C}++$ that executes a state machine consisting of three sections: a modem initialization section, a TDMA sequence of commands, and a clock watchdog.

Acoustic modem drivers previously reported by the authors and others for synchronous navigation, such as the modem drivers employed on the MIT autonomous surface vehicles, [3], and the WHOI Seabed vehicles, [4], [6], are not portable due to the tight integration of these modem drivers into the vehicle-specific application code of their respective vehicle control and navigation systems. In contrast, the Acomms software reported herein is portable, employs a vehicle-independent interface based on UDP messages, runs 
TABLE I

TDMA CYCLE COMMAND SUMMARY

\begin{tabular}{c||c||c}
\hline Command & Size & Addt'l Info \\
\hline \hline Configuration & n/a & supports all available cfgs \\
\hline Ranging Ping & 32 bits & return OWTT between nodes \\
\hline Mini-Packet & 32 bits & user specified codes (Abort) \\
\hline Cycle-Init & 32 bits & initiates data tx \\
\hline Data Packet & $32-2048$ bytes & varies by encoding type \\
\hline LBL & n/a & listen on 4 freq \\
\hline PAUSE & n/a & fixed length pause \\
\hline VLPAUSE & n/a & variable length pause \\
\hline
\end{tabular}

as a stand-alone daemon on a host Linux CPU, and operates symmetrically on all node types - e.g. underwater vehicles, fixed beacons, and surface ships.

The Acomms software is designed to act as a transport layer between the host computer and the modem, passing through all message traffic in both directions. All communications with the modem as well as various statistics on messages transmitted and received are time-stamped and logged. In addition, the Acomms software enables synchronous communication and navigation as described below.

The Acomms software supports a user-configured TDMA cycle of modem commands that is executed continuously except when interrupted by the modem initialization process or the clock watchdog. The TDMA cycle is used to command the modem to transmit acoustic messages, interrogate long baseline (LBL) beacons or change selective modem configurations such as the transmission frequency and bandwidth on the fly. Table I shows the TDMA commands currently available in the Acomms software.

In addition to all of the capabilities that are applicable to asynchronous communication, the Acomms system enables synchronized communication and OWTT navigation when equipped with a precision time receiver. The Acomms software supports OWTT navigation through a message packing function that precisely controls the timing of when a message is provided to the modem as specified in [12] and can thus properly anticipate and encode the time-of-launch of the data packet. The Acomms software also ensures that the modem's internal clock, which is used to measure the time-of-arrival of messages, is properly disciplined.

\section{DeCEntralized Extended Information Filter}

Decentralized estimation in the context of underwater communication and navigation faces unique constraints in terms of low bandwidth and high latency, which renders many of the decentralized estimation solutions from land-based applications unsuitable. Until recently little research has been done on the topic of decentralized estimators and multi-vehicle navigation in the field of underwater robotics. However, as the cost of vehicles has decreased and their reliability improved, the increased interest in multi-vehicle operations within the ocean science community has precipitated new research in this area. In [2] the author presents a method that allows a vehicle running a Bayes estimator to use range and position information broadcast from one or more moving beacons. This work expands on the moving long baseline concept in [24] to encompass multiple range sources and real-time operation. The authors of [19] address a similar concept to moving long baseline and compare the use of the Kalman filter to a particle filter on the vehicle's localization performance.

This section describes a decentralized extended information filter (DEIF) designed for one-way-travel-time navigation [28]. The implementation of the DEIF relies on two separate filters, both of which process sensor data causally and asynchronously. The information filter on the ship has access to ship sensor data but not range measurements (we will use the term ship to refer to the reference beacon for the remainder of this derivation). The ship-based filter is used to calculate the change in the ship's information vector and information matrix between acoustic broadcasts, and this delta information is acoustically transmitted to the vehicle. The DEIF on the vehicle is designed to run locally on a submerged vehicle with real-time access to the vehicle's navigation sensors and infrequent, asynchronous access to acoustic broadcasts from a moving reference beacon. The DEIF does not have access to real-time global positioning system (GPS) measurements from the reference beacon or any other information except information that is received acoustically. Figure 2 shows a schematic of the delta ship information transmitted from the ship to the vehicle, where TOL refers to the time-of-launch of an acoustic data packet, and TOA refers to the time-of-arrival.

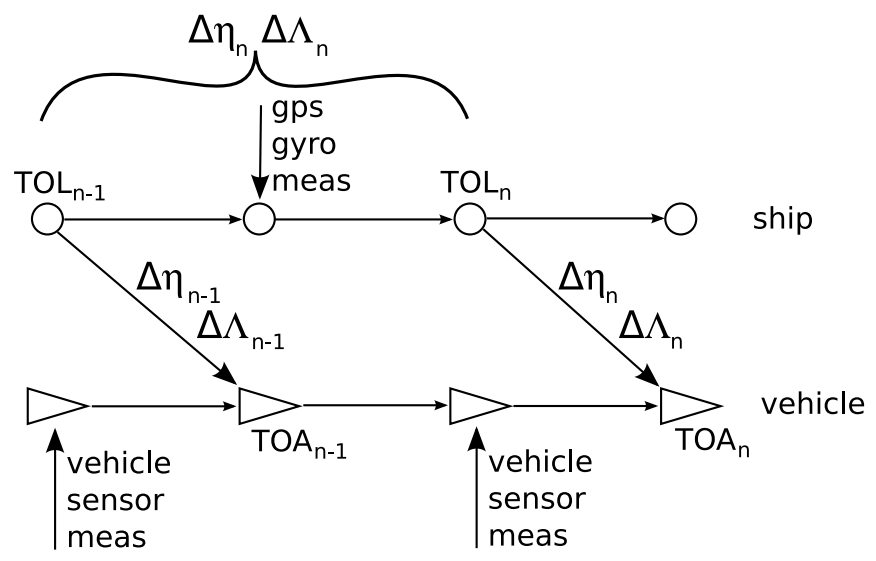

Fig. 2. A schematic of the information contained in the range packet acoustically transmitted from the ship to the vehicle.

The estimation algorithm that most closely resembles the DEIF is [7], where the authors rely on a single moving georeferenced beacon to support the localization of multiple vehicles through asynchronous acoustic broadcasts. The main difference between the algorithm presented in [7] and our DEIF algorithm is that [7] employs a vehicle-based EKF and performs range measurement updates using the absolute position and covariance broadcast from the reference beacon. The benefit of this formulation is that the algorithm is trivially robust to packet loss, however excluding the reference beacon position from the state vector of the filter makes the results 
difficult or impossible to compare analytically to a centralized filter. In contrast, the decentralized algorithm reported herein recreates the results of a centralized extended Kalman filter (CEKF) that has real-time access to measurements from both the vehicle and the beacon's navigation sensors. In addition, by keeping both vehicle and ship states in the filter, range measurement updates do not risk over confidence in and correlation of observations. In [2], the work upon which [7] is based, a multi-hypothesis strategy is employed to avoid over-confidence by preventing measurement data from being incorporated multiple times. To the best knowledge of the authors, the DEIF, presented in detail and with derivations in [25], [28], is the first formulation of an extended information filter in the context of decentralized single-beacon navigation for underwater vehicles.

\section{A. Information Filter Equations}

The EIF is characterized by the information matrix, $\boldsymbol{\Lambda}$, and the information vector, $\boldsymbol{\eta}$, which can be defined in terms of the mean, $\boldsymbol{\mu}$, and covariance, $\boldsymbol{\Sigma}$, of the state vector, $\boldsymbol{x},[11]$, [20], as

$$
\begin{aligned}
& \boldsymbol{\Lambda}=\boldsymbol{\Sigma}^{-1} \\
& \boldsymbol{\eta}=\boldsymbol{\Lambda} \boldsymbol{\mu}
\end{aligned}
$$

where

$$
\begin{aligned}
\boldsymbol{\mu} & =E[\boldsymbol{x}] \\
\boldsymbol{\Sigma} & =E\left[(\boldsymbol{x}-\boldsymbol{\mu})(\boldsymbol{x}-\boldsymbol{\mu})^{\top}\right] .
\end{aligned}
$$

\section{B. Ship-Based Information Filter}

We assume a constant-velocity linear kinematic process model for the ship, which, in the experience of the authors, is a reasonable assumption for ship motion in the context of AUV survey operations. For the ship's process model the state vector contains the ship's xy-position, heading, and the respective velocities,

$$
\boldsymbol{x}_{s}=\left[x_{s}, y_{s}, \theta_{s}, \dot{x}_{s}, \dot{y}_{s}, \dot{\theta}_{s}\right]^{\top} .
$$

The ship process model is identical to that reported in [27], to which the reader is referred for further details.

What differentiates the DEIF from other estimators used in decentralized single-beacon navigation is the information that is transmitted with the range measurements and how that information is incorporated into the decentralized vehicle navigation filter in conjunction with the range measurement. To initiate a range measurement, the ship broadcasts an acoustic data packet, or range packet, containing information about the ship state. In other formulations of single-beacon navigation, such as [2] and [7], the range packet contains the mean and covariance of the ship's current $\mathrm{x}-\mathrm{y}$ position, which is used by the filter on the vehicle to perform a range measurement update. This approach, while it has certain advantages, does not contain enough information to allow the vehicle to recreate the global state of the system, including full state recovery of both the vehicle and the ship.

In the DEIF formulation, the range packet contains the change in $\boldsymbol{\Lambda}_{s}$ and $\boldsymbol{\eta}_{s}$ between the time of the current acoustic broadcast, $\mathrm{TOL}_{n}$, and the time of the previous acoustic broadcast, $\mathrm{TOL}_{n-1}$,

$$
\begin{aligned}
\Delta \boldsymbol{\Lambda}_{T O L_{n}} & =\boldsymbol{\Lambda}_{s_{T O L_{n}}}-\boldsymbol{\Lambda}_{s_{T O L_{n-1}}} \\
\Delta \boldsymbol{\eta}_{T O L_{n}} & =\boldsymbol{\eta}_{s_{T O L_{n}}}-\boldsymbol{\eta}_{s_{T O L_{n-1}}}
\end{aligned}
$$

where, for conformability, $\boldsymbol{\Lambda}_{s_{T O L_{n-1}}}$ and $\boldsymbol{\eta}_{s_{T O L_{n-1}}}$ have been padded with zeros to match the size of $\boldsymbol{\Lambda}_{s_{T O L_{n}}}$ and $\boldsymbol{\eta}_{s_{T O L_{n}}}$ respectively. These range packets are reassembled subsea in the DEIF.

\section{Vehicle-Based Decentralized Extended Information Filter}

The DEIF uses a constant velocity nonlinear process model with a 12 degree-of-freedom (DOF) state vector,

$$
\boldsymbol{x}_{v}=\left[\boldsymbol{s}^{\top}, \boldsymbol{\varphi}^{\top}, \boldsymbol{v}^{\top}, \boldsymbol{\omega}^{\top}\right]^{\top}
$$

where $s$ is the local-level vehicle pose in the local frame, $\varphi$ is the local-level vehicle attitude (Euler roll, pitch, heading), $\boldsymbol{v}$ is the body-frame linear velocity, and $\boldsymbol{\omega}$ is the body-frame angular velocity. The vehicle process model is identical to that reported in [27], to which the reader is referred for further details.

1) DEIF State Vector: In addition to the current vehicle state, the DEIF maintains a copy of the historic ship states reconstructed from the delta information acoustically broadcast from the ship. As a result, the DEIF state vector consists of two parts, the current vehicle state and the historic ship states,

$$
\boldsymbol{x}_{v_{k \mid k}}=\left[\begin{array}{c}
x_{v_{k}} \\
x_{s_{T O L_{n}}} \\
\vdots \\
x_{s_{T O L_{1}}}
\end{array}\right]
$$

where we adopt the convention that $\boldsymbol{x}_{v_{k \mid k}}$ denotes the entire vehicle state vector at time $k ; x_{v_{k}}$ is the current vehicle state; and $x_{s_{T O L_{n}}}$ is the ship state when the $\mathrm{n}^{\text {th }}$ range packet was broadcast.

2) Incorporating Delta Ship Information: At the time-ofarrival (TOA) of a range packet at the vehicle, the delta ship information included in each range packet is incorporated in the DEIF, and then the range measurement update is performed. The delta ship information is incorporated into the DEIF simply by addition, in the analogous operation to (6) and (7):

$$
\begin{aligned}
\boldsymbol{\Lambda}_{v_{T O A_{n}}} & =\overline{\boldsymbol{\Lambda}}_{v_{T O A_{n}}}+\Delta \boldsymbol{\Lambda}_{T O L_{n}} \\
\boldsymbol{\eta}_{v_{T O A_{n}}} & =\overline{\boldsymbol{\eta}}_{v_{T O A_{n}}}+\Delta \boldsymbol{\eta}_{T O L_{n}}
\end{aligned}
$$

where $\bar{\Lambda}_{v_{T O A_{n}}}$ is the information matrix before the delta ship information is incorporated and $\boldsymbol{\Lambda}_{v_{T O A_{n}}}$ is the information matrix after the delta ship information is incorporated, accounting for conformability. As noted in Section IV-B, the delta ship information encapsulates all of the information that the filter has gained about the ship state since the last range packet was transmitted. The simplicity of this computation is one of the advantages of the information filter. 
3) Range Measurement Updates: At the time-of-arrival of the range packet, after the delta ship information is incorporated, the range measurement update is performed. The observation model for the range measurement is

$$
z_{k}=\sqrt{\left(\boldsymbol{x}_{v_{x y z}}-\boldsymbol{x}_{s_{x y z}}\right)^{\top}\left(\boldsymbol{x}_{v_{x y z}}-\boldsymbol{x}_{s_{x y z}}\right)}+v_{k}
$$

where $\boldsymbol{x}_{v_{x y z}}$ is the vehicle pose at the TOA, $\boldsymbol{x}_{s_{x y z}}$ is the ship pose at the TOL, and $v_{k} \sim \mathcal{N}\left(0, R_{k}\right)$.

Due to the cumulative nature of this navigation technique, packet loss is an operational concern for real-time implementation. The authors are currently investigating several viable options for addressing the effects of packet loss, including broadcasting redundant range packets or reformulating the delta information in order to mitigate the effect of packet loss.

4) Recreating Centralized Results: As stated above, we use the CEKF as the benchmark for the performance of the DEIF. The contribution to $\Delta \boldsymbol{\Lambda}_{T O L_{n}}$ and $\Delta \boldsymbol{\eta}_{T O L_{n}}$ from the ship sensor measurement updates are independent of other measurements because of the additive nature of the measurement update in the information filter and the linear ship process model. As a result, the $\Delta \boldsymbol{\Lambda}_{T O L_{n}}$ and $\Delta \boldsymbol{\eta}_{T O L_{n}}$ calculated from the ship-based filter, which has no knowledge of the vehicle and is not subject to range measurement updates, is identical to what the DEIF would have calculated if it was performing the ship process predictions and ship sensor measurement updates even though the ship states in the DEIF have been modified by prior range measurements. Thus at the instant of each range measurement the DEIF exactly recreates the results of the CEKF. Between range measurements, the CEKF and DEIF estimates of the vehicle's state will not be identical because of linearization errors.

\section{Simulation Results}

The DEIF is tested using a simulated 6 hours survey at $3800 \mathrm{~m}$ depth. To test the validity of the filter, we compare the DEIF estimation results to those obtained with a CEKF, reported in [27], at every time step. Note that the results from the CEKF with experimentally collected navigation data were used by the authors in [27] to validate OWTT navigation as a viable alternative to tradition navigation techniques. The simulation results presented here are intended to validate the claim that the DEIF can recreate the results of the CEKF.

\section{A. Simulation Setup}

For comparison purposes this simulation is designed to mimic the experimental setup of the deep water survey [27]. In the simulated mission presented here, the vehicle drives ten $700 \mathrm{~m}$ tracklines spaced $80 \mathrm{~m}$ apart at a velocity of 0.35 $\mathrm{m} / \mathrm{s}$. The vehicle's depth is constant at $3800 \mathrm{~m}$. The vehicle takes approximately 6 hours to complete the survey, during which time the ship drives around the vehicle's survey area in a diamond pattern at $0.5 \mathrm{~m} / \mathrm{s}$, broadcasting acoustic data packets every 2.5 minutes.

We assume that the ship is equipped with a differential global positioning system (DGPS) receiver and a gyrocompass to measure heading. The vehicle has an OCTANS fiber-optic
TABLE II

SIMULATED NAVIGATION SENSORS: SAMPLING FREQUENCY AND NOISE

\begin{tabular}{l|l|l}
\hline \hline Vehicle Sensors & Frequency & Noise \\
\hline & & $h: 0.1^{\circ}$ \\
& & $r p: 0.01^{\circ}$ \\
OCTANS $^{a}$ & $3 \mathrm{~Hz}$ & $0.5^{\circ} / \mathrm{s}$ \\
& & $\dot{r} \dot{p}: 0.25^{\circ} / \mathrm{s}$ \\
\hline depth sensor & $0.9 \mathrm{~Hz}$ & $5 \mathrm{~cm}$ \\
\hline DVL & $3.0 \mathrm{~Hz}$ & $1 \mathrm{~cm} / \mathrm{s}$ \\
\hline modem & every $2.5 \mathrm{~min}$ & $4 \mathrm{~m}$ \\
\hline \hline
\end{tabular}

${ }^{a} h, p$, and $r$ are heading, pitch, and roll respectively; $\dot{h}, \dot{p}$, and $\dot{r}$ are heading, pitch, and roll rates.

\begin{tabular}{l|l|l}
\hline \hline Ship Sensors & Frequency & Noise \\
\hline GPS & $1.0 \mathrm{~Hz}$ & $0.5 \mathrm{~m}$ \\
\hline gyrocompass & $2.0 \mathrm{~Hz}$ & $0.1^{\circ}$ \\
\hline \hline
\end{tabular}

gyrocompass to measure attitude and attitude rates; a Paroscientific pressure sensor to measure depth; and an RDI Doppler velocity $\log$ (DVL) to measure bottom-referenced velocities. Acoustic modems are used to measure the range between the ship and the vehicle. The vehicle and ship navigation sensors, their sampling frequencies, and the noise statistics for each sensor are given in Table II.

\section{B. Results}

The results of the simulation are shown in Figures 3, 4 and 5. Figure 3 shows the estimated vehicle trajectory overlaid with the 3-sigma covariance of the vehicle position as estimated by the DEIF. The GPS-reported position of the ship as it moves around the vehicle survey area is also shown. Figure 4 shows the difference between the vehicle's true position and the estimate from the DEIF of the vehicle's position over the course of the simulated dive. The error at the end of the dive between the DEIF's estimate of the vehicle position and the true vehicle position is $3.7 \mathrm{~m}$ cross-track and $0.2 \mathrm{~m}$ alongtrack both with $3.1 \mathrm{~m}$ standard deviation. For comparison, had the vehicle relied solely on dead reckoning throughout the dive with no range measurements, the error at the end of the dive between the estimated and true vehicle position would have been $8.8 \mathrm{~m}$ cross-track and $5.6 \mathrm{~m}$ along-track with a 7.8 $\mathrm{m}$ standard deviation. The 3 -sigma error bars are included to show that the filter maintains consistency over the course of the dive.

Comparing the mean of the vehicle's 12 degree-of-freedom (DOF) state vector as estimated by the DEIF versus the CEKF, Figure 5 shows the norm of the difference over the course of the simulation. The lower plot highlights the norm of the difference immediately after a range measurement, as marked by the asterisks. Note that the $y$-axis on the lower plot has been scaled down by two orders of magnitude to show the precision with which the DEIF is able to reproduce the results of the CEKF. The average difference between the filters across the entire dive is $5.68 \mathrm{e}-3(5.7 \mathrm{~mm})$ in $\mathrm{x}-\mathrm{y}$ position and $3.35 \mathrm{e}-8$ in the other state elements. The average difference immediately after a range measurement is $8.27 \mathrm{e}-5 \mathrm{~m}$ in $\mathrm{x}-\mathrm{y}$ position and 
$1.70 \mathrm{e}-10$ in the other vehicle states. These results support the prediction based on the theory that the DEIF produces state estimates that are comparable to the CEKF: immediately after each range update the results should be identical within the tolerance of numerical precision; between range updates, the results should differ only due to linearization errors. Over the course of the 6 hour simulated dive the difference in $\mathrm{x}$ $y$ position between the DEIF and the EKF is, on average, 8.27e-5 $\mathrm{m}$ immediately after each range update. In addition, the difference between the filters due to linearization errors (averaged over the entire dive) is $5.7 \mathrm{~mm}$ on average with a maximum difference of $4.9 \mathrm{~cm}$.

Because the results presented here are based on a simulated data set, there are several possible discrepancies compared to experimental data. The assumed noise characteristics of the navigation sensors in Table II are used both in the simulation of noisy sensor data and in the measurement models in the DEIF and CEKF. As a result the measurement models exactly model the performance of the navigation sensors. In addition, the noise model of every sensor is assumed to be Gaussian. While these assumptions may be reasonable for common vehicle navigation sensors that have been tested extensively in the field [15], acoustic range measurements suffer from highlyvariable, non-Gaussian noise sources including multi-path and ray-bending errors. In an attempt to account for this, we use a large assumed variance for the range measurements. A realworld solution would require outlier filtering of the range data.

\section{Vi. CONCLUSIONS}

The Acomms system has been successfully installed on three AUVs and utilized to manage all acoustic communications during four oceanographic expeditions.

The structure of the information filter makes it a natural choice for a decentralized implementation. Delayed ship up-

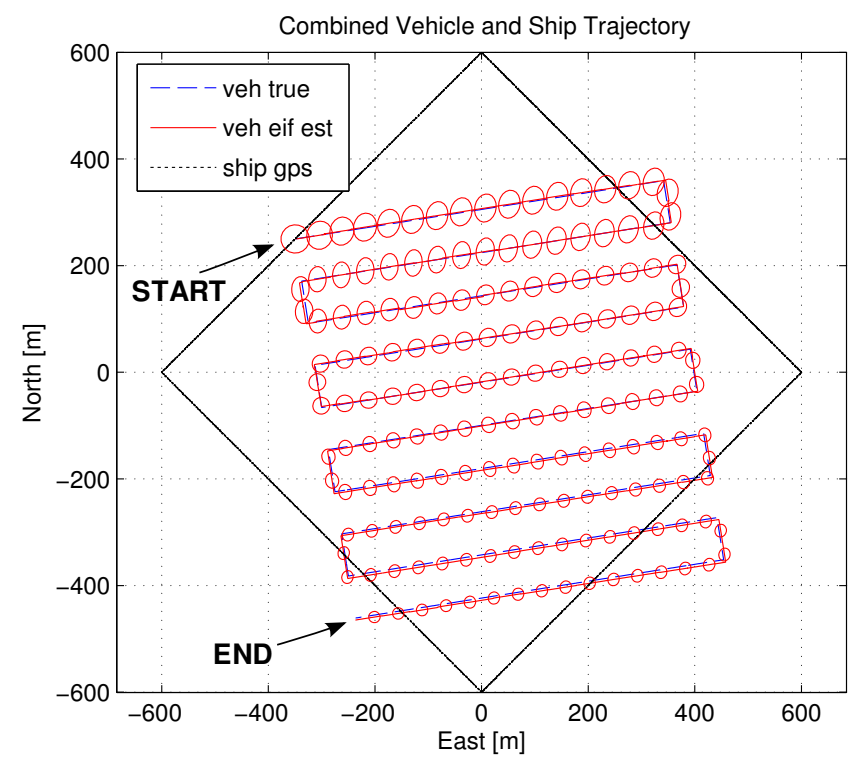

Fig. 3. Ship and vehicle trajectories. The vehicle started in the northwest and proceeded east, ending at the southwest corner of the survey. The ship moved counter-clockwise around the diamond starting at the eastern-most apex.
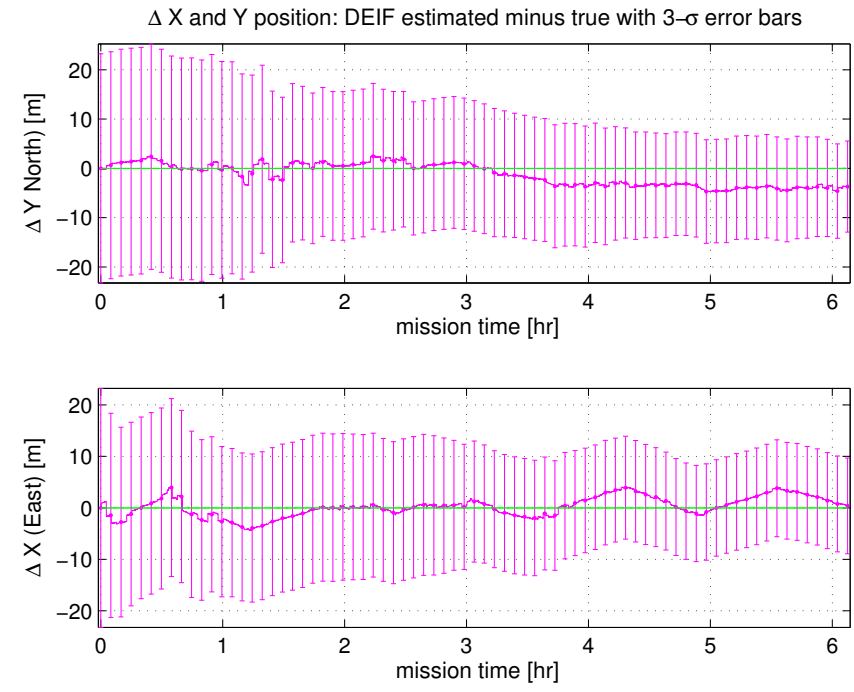

Fig. 4. The difference between the true vehicle position and estimate from the DEIF over time. The error bars are 3 times the standard deviation in each degree of freedom.

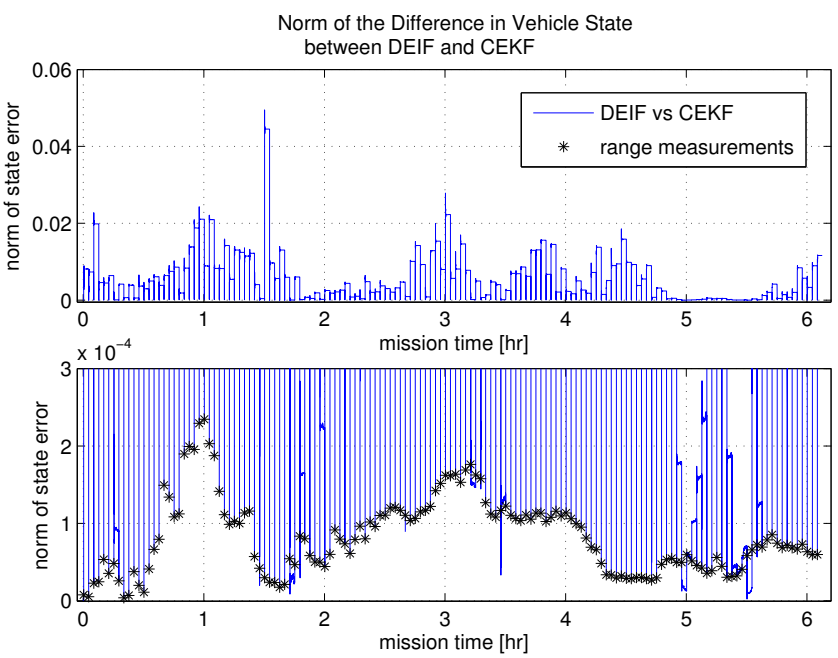

Fig. 5. The sum of the squared error between the mean vehicle position as estimated by the DEIF versus the CEKF.

dates are simply additive and require a minimal amount of information to be acoustically transmitted that is well within the function limits of available acoustic modems [8], [9].

In this paper we have described the structure and implementation of the acoustic communications (Acomms) system that has been successfully installed on three AUVs and utilized to manage all acoustic communications during four oceanographic expeditions. In addition we have described a vehiclebased extended information filter that is able to estimate a vehicle's state, including $x-y$ position, using only vehiclebased inertial navigation sensors and asynchronous acoustic broadcasts from a single, moving, georeferenced beacon. The DEIF is able to locally recreate vehicle state estimates that are commensurate with the results from a centralized extended Kalman filter within a margin of numerical error, and did 
so over the course of a simulation that is representative of an actual, deep-water survey in both physical scale and the frequency of measurements. In addition, the filter in its current form could be used on multiple underwater vehicles where each vehicle simultaneously receives acoustic data broadcasts from the reference beacon. Given the favorable results in simulation of the DEIF, we look forward to experimentally validating this algorithm and continuing to work towards a full multi-vehicle implementation.

In the future, the natural expansion of this algorithm is to incorporate acoustic broadcasts from other vehicles in addition to broadcasts from the reference beacon. The addition of vehicle-based acoustic broadcasts would generate inter-vehicle range information that could be used to further constrain each receiving vehicle's navigation solution. Incorporating intervehicle ranges presents a number of challenges for continued research, including the nonlinearity of the process models of the vehicles initiating the acoustic broadcast, and the problem of over confidence associated with double counting information passed between the vehicles.

\section{ACKNOWLEDGMENTS}

The authors are grateful for Micro-Modem support from current and former members of the WHOI Acoustic Communications Group including Keenan Ball, Lee Freitag, Matthew Grund, and Sandipa Singh. We are grateful to Clay Kunz and Giancarlo Troni for their help daemonizing the Acomms software. The authors would also like to thank Chris Taylor, John Bailey, and the entire Nereus team for their help implementing the hardware for the Acomms system on Nereus. Some of the results reported herein were previously reported in [26], [28].

This work was supported by the National Science Foundation under NSF awards ATM-0427220, ATM-0428122, and IIS-0746455. The Nereus project was supported by the National Science Foundation under award OCE-0334411, the Office of Naval Research under work order N0001409WX20051, the National Oceanic and Atmospheric Administration under award NA04OAR4300168, the Woods Hole Oceanographic Institution, and the Russell Family Foundation.

\section{REFERENCES}

[1] P. Baccou and B. Jouvencel, "Homing and navigation using one transponder for AUV, postprocessing comparisons results with long base-line navigation," in Proc. IEEE Int. Conf. Robot. Auto. (ICRA), vol. 4, 2002, pp. 4004-4009.

[2] A. Bahr, "Cooperative localization for autonomous underwater vehicles," Ph.D. dissertation, Massachusetts Institute of Technology, Cambridge, MA, USA, February 2009.

[3] J. Curcio, J. Leonard, J. Vaganay, A. Patrikalakis, A. Bahr, D. Battle H. Schmidt, and M. Grund, "Experiments in moving baseline navigation using autonomous surface craft," in Proc. IEEE/MTS OCEANS Conf. Exhib., Washington, D.C., Sep. 2005, pp. 730-735 Vol. 1.

[4] R. M. Eustice, L. L. Whitcomb, H. Singh, and M. Grund, "Recent advances in synchronous-clock one-way-travel-time acoustic navigation," in Proc. IEEE/MTS OCEANS Conf. Exhib., Boston, MA, USA, Sep. 2006, pp. 1-6.

[5] R. M. Eustice, H. C. Brown, and A. Kim, "An overview of auv algorithms research and testbed at the university of michigan," in IEEE/OES AUV2008 Conference on Polar AUVs, Woods Hole, MA, 2008, pp. 1-9, in Press.

[6] R. M. Eustice, L. L. Whitcomb, H. Singh, and M. Grund, "Experimental results in synchronous-clock one-way-travel-time acoustic navigation for autonomous underwater vehicles," in Proc. IEEE Int. Conf. Robot. Auto. (ICRA), Rome, Italy, Apr. 2007, pp. 4257-4264.
[7] M. F. Fallon, G. Papadopoulos, and J. J. Leonard, "Cooperative AUV navigation using a single surface craft," in Proc. of Field and Service Robotics, Cambridge, MA, Jul 2009.

[8] L. Freitag, M. Grund, J. Partan, K. Ball, S. Singh, and P. Koski, "Multiband acoustic modem for the communications and navigation aid AUV," in Proc. IEEE/MTS OCEANS Conf. Exhib., Washington, D.C., Sep. 2005, pp. 1080-1085.

[9] L. Freitag, M. Grund, S. Singh, J. Partan, P. Koski, and K. Ball, "The WHOI micro-modem: an acoustic communications and navigation system for multiple platforms," in Proc. IEEE/MTS OCEANS Conf. Exhib., Washington, D.C., Sep. 2005, pp. 1086-1092.

[10] A. Gadre and D. Stilwell, "A complete solution to underwater navigation in the presence of unknown currents based on range measurements from a single location," in Proc. IEEE/RSJ Intl. Conf. Intell. Robots Systems (IROS), Edmonton AB, Canada, 2005, pp. 1420-1425.

[11] A. Gelb, Ed., Applied optimal estimation. Cambridge, MA: MIT Press, 1982.

[12] M. Grund, J. Partan, P. Koski, and L. Freitag, "Synchronous navigation with the micro-modem," WHOI, Tech. Rep., 2005, document Revision: D.

[13] M. Hunt, W. Marquet, D. Moller, K. Peal, W. Smith, and R. Spindel, "An acoustic navigation system," Woods Hole Oceanographic Institution, Tech. Rep. WHOI-74-6, Dec. 1974.

[14] D. Kilfoyle and A. Baggeroer, "The state of the art in underwater acoustic telemetry," Oceanic Engineering, IEEE Journal of, vol. 25, no. 1, pp. 4-27, Jan 2000.

[15] J. C. Kinsey, R. M. Eustice, and L. L. Whitcomb, "A survey of underwater vehicle navigation: recent advances and new challenges," in IFAC Conf. on Manoeuvring and Control of Marine Craft, Lisbon, Portugal, Sep. 2006.

[16] M. B. Larsen, "Autonomous navigation of underwater vehicles," $\mathrm{Ph} . \mathrm{D}$. dissertation, Technical University of Denmark, Denmark, Feb. 2001.

[17] S. McPhail and M. Pebody, "Range-only positioning of a deep-diving autonomous underwater vehicle from a surface ship," Oceanic Engineering, IEEE Journal of, vol. 34, no. 4, pp. 669-677, Oct. 2009.

[18] Meinberg LANTIME/GPS Manual: EXT 1HE V4, Meinberg Funkuhren GmbH \& Co. KG, Bad Pyrmont, Germany, May 2004.

[19] D. Mirabello, A. C. Sanderson, and D. R. Blidberg, "Comparing Kalman and particle filter approaches to coordinated multi-vehicle navigation," in Proc. Intl. Symp. Unmanned Untethered Subm. Tech. (UUST), Aug. 2007.

[20] A. G. O. Mutambara, Decentralized Estimation and Control for Multisensor Systems. Boca Raton, FL, USA: CRC Press, Inc., 1998.

[21] A. Scherbatyuk, "The AUV positioning using ranges from one transponder LBL," in Proc. IEEE/MTS OCEANS Conf. Exhib., vol. 3, San Diego, California, 1995, pp. 1620-1623.

[22] S. Singh, M. Grund, B. Bingham, R. M. Eustice, H. Singh, and L. Freitag, "Underwater acoustic navigation with the WHOI micromodem," in Proc. IEEE/MTS OCEANS Conf. Exhib., Boston, MA, Sep. 2006.

[23] S. Singh, S. E. Webster, L. Freitag, L. L. Whitcomb, K. Ball, J. Bailey, and C. Taylor, "Acoustic communication performance of the WHOI micro-modem in sea trials of the Nereus vehicle to $11,000 \mathrm{~m}$ depth," in Proc. IEEE/MTS OCEANS Conf. Exhib., Biloxi, MS, Oct 2009, In Press.

[24] J. Vaganay, J. Leonard, J. Curcio, and J. Willcox, "Experimental validation of the moving long base-line navigation concept," in Autonomous Underwater Vehicles, 2004 IEEE/OES, June 2004, pp. 59-65.

[25] S. E. Webster, "Decentralized single-beacon acoustic navigation: Combined communication and navigation for underwater vehicles," $\mathrm{Ph}$.D. dissertation, Johns Hopkins University, Baltimore, MD, USA, June 2010.

[26] S. E. Webster, R. M. Eustice, C. Murphy, H. Singh, and L. L. Whitcomb, "Toward a platform-independent acoustic communications and navigation system for underwater vehicles," in Proc. IEEE/MTS OCEANS Conf. Exhib., Biloxi, MS, Oct 2009, In Press.

[27] S. E. Webster, R. M. Eustice, H. Singh, and L. L. Whitcomb, "Preliminary deep water results in single-beacon one-way-travel-time acoustic navigation for underwater vehicles," in Proc. IEEE/RSJ Intl. Conf. Intell. Robots Systems (IROS), St. Louis, MO, Oct. 2009, pp. 2053-2060.

[28] S. E. Webster, L. L. Whitcomb, and R. M. Eustice, "Preliminary results in decentralized estimation for single-beacon acoustic underwater navigation," in Proc. Robotics: Sci. \& Sys. Conf., Zaragoza, Spain, June 2010, Accepted, To Appear. 